\title{
Correction to: MY01D binds with kinase domain of the EGFR family to anchor them to plasma membrane before their activation and contributes carcinogenesis
}

Yoo-Seung Ko · Jeong A. Bae - Keon Young Kim (1) - Sung Jin Kim · Eun Gene Sun · Kyung Hwa Lee • Nacksung Kim · Hyuno Kang · Young-Woo Seo · Hangun Kim • Ik Joo Chung $\cdot$ Kyung Keun Kim

Published online: 21 April 2021

(c) The Author(s), under exclusive licence to Springer Nature Limited 2021

\section{Correction to: Oncogene}

https://doi.org/10.1038/s41388-019-0954-8

Following the publication of this article, the authors noted three invasion images in Fig. 7 were repeated; the two images in Fig. 7a (EV, WT) and one image in Fig. 7b (TH) were repeated in Fig. 7c $(\mathrm{EV}, \mathrm{WT}, \mathrm{TH})$. The corrected Fig. 7 is shown below. The authors would like to apologize for any inconvenience caused.

The original article has been corrected. 
a
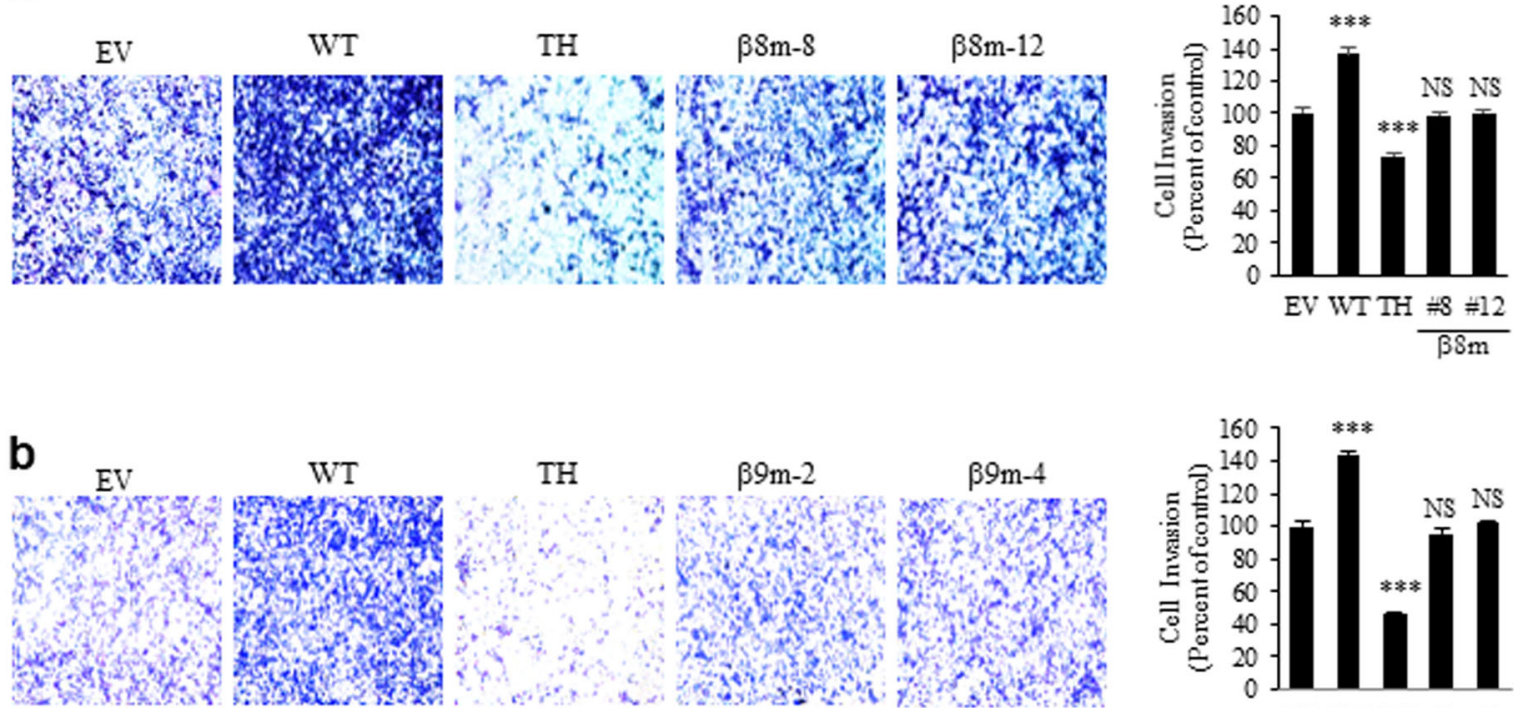

$\mathrm{TH}$
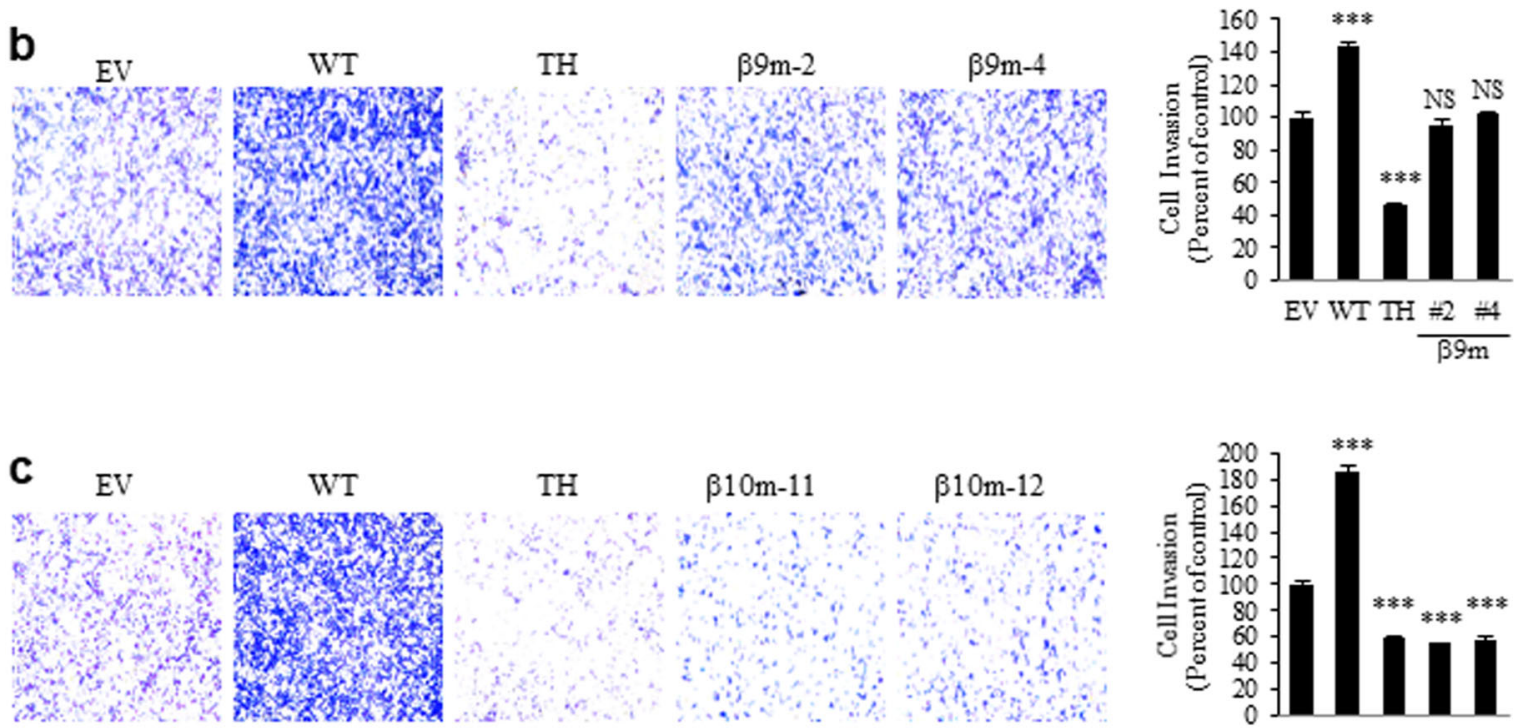

$\mathrm{TH}$
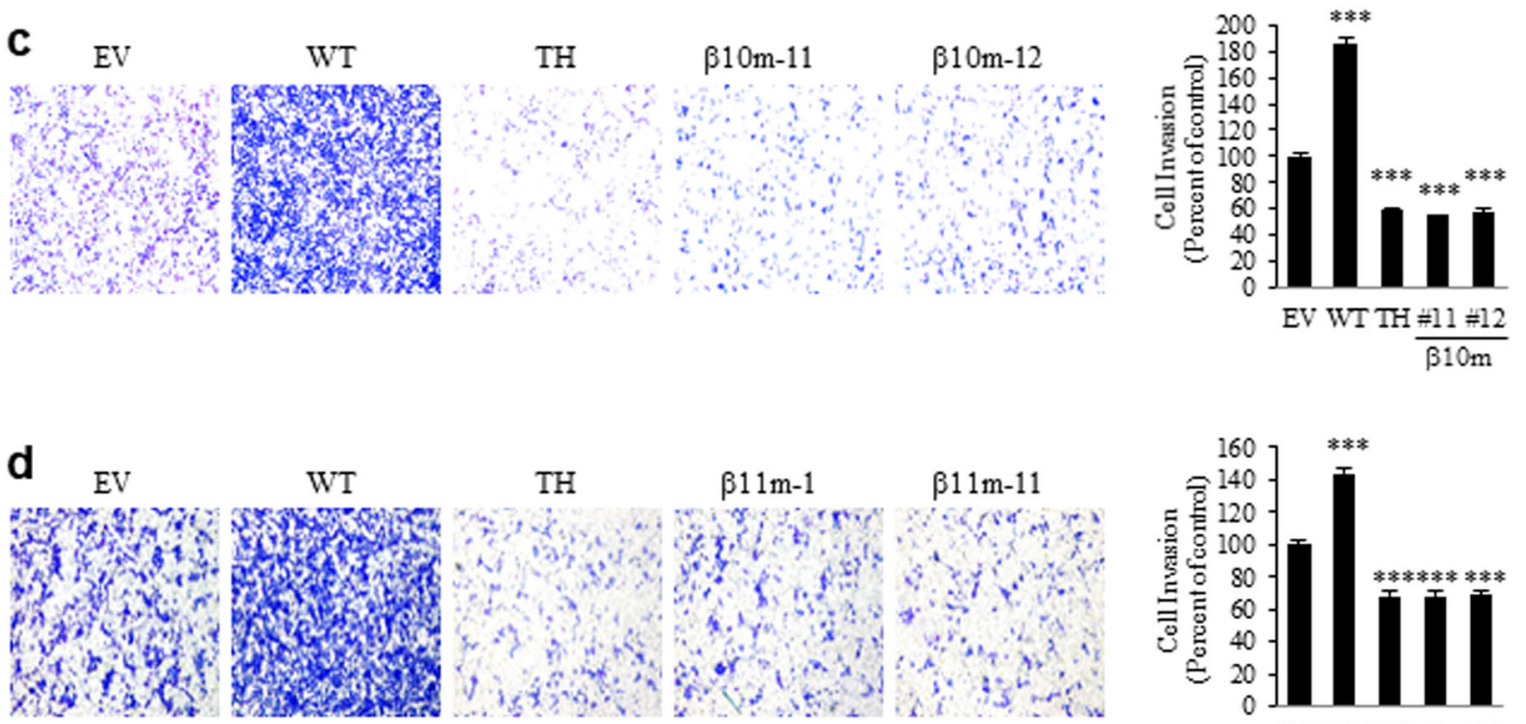

$\beta 11 \mathrm{~m}-1$
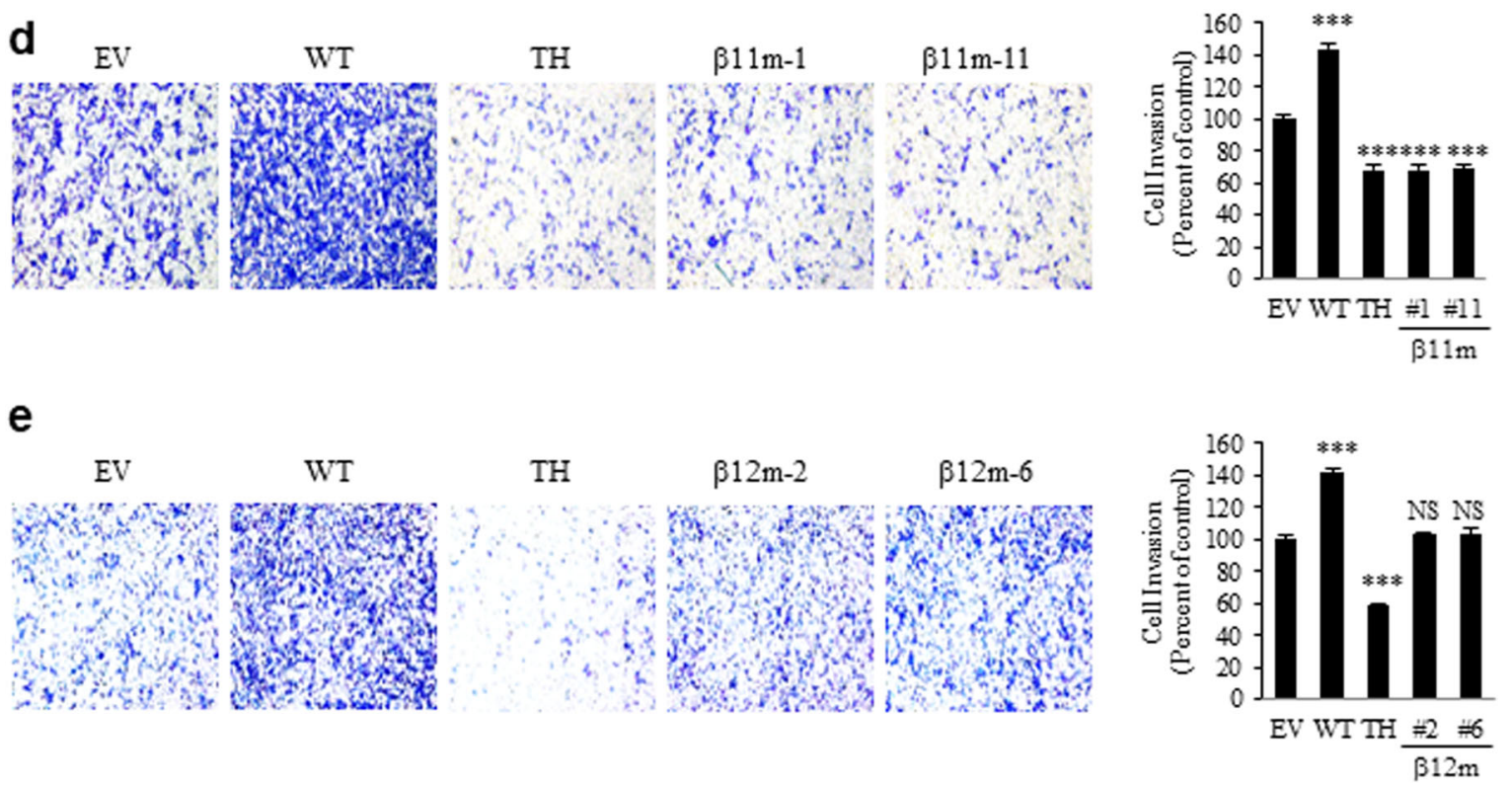

Fig. 7 Three $\beta$-sheets in the C-terminal end of MYO1D (except for $\beta 10$ and $\beta 11)$ are essential to the RTK-binding site. Caco2 cells were co-transfected with empty vector, MYO1D WT, MYO1D TH1 domain $(\mathrm{TH})$, or the mutated TH1 construct $(\beta 8 \mathrm{~m}, \beta 9 \mathrm{~m}, \beta 10 \mathrm{~m}, \beta 11 \mathrm{~m}$, or $\beta 12 \mathrm{~m}$ ) for $48 \mathrm{~h}$ and subjected to the transwell invasion assay. $\beta 8 \mathrm{~m}$ was made by substitution the residues of 8 th $\beta$-sheet at a time by alanine (Table S2), leaving the remaining $\beta$-sheets $(\beta 9, \beta 10, \beta 11$, and $\beta 12$ ) intact. The expression of each mutated TH1 construct was confirmed in lysates from the same cells used for the invasion assay (right side). The pictures and histogram of the invasion assay were obtained as in Fig. 1c. 